\title{
Some qualitative properties of nonlinear fractional integro-differential equations of variable order
}

\author{
Ahmed Refice $^{a}$, Mohammed Said Souid ${ }^{b}$ and Ali Yakar ${ }^{c^{*}}$ \\ a Laboratory of Mathematics, Djillali Liabes University of Sidi Bel-Abbès, Algeria \\ ${ }^{b}$ Department of Economic Sciences, University of Tiaret, Algeria \\ c Department of Mathematics, Gaziosmanpasa University, Tokat, Turkey \\ ahmedrefice@gmail.com,souimed2008@yahoo.com,ali.yakar@gop.edu.tr
}

\section{ARTICLE INFO}

Article History:

Received 25 November 2021

Accepted 30 December 2021

Available 31 December 2021

\section{Keywords:}

Fractional differential equations

of variable order

Boundary value problem

Fixed point theorem

Ulam-Hyers-Rassias stability

AMS Classification 2010:

45J05; 34D20; 26A33; $34 A 08$

\begin{abstract}
The existence-uniqueness criteria of nonlinear fractional integro-differential equations of variable order with multiterm boundary value conditions are considered in this work. By utilizing the concepts of generalized intervals combined with the piecewise constant functions, we transform our problem into usual Caputo's fractional differential equations of constant order. We develop the necessary criteria for assuring the solution's existence and uniqueness by applying Schauder and Banach fixed point theorem. We also examine the stability of the derived solution in the Ulam-Hyers-Rassias (UHR) sense and provide an example to demonstrate the credibility of the results.
\end{abstract}

\section{Introduction}

The fundamental idea behind fractional calculus is simply to replace the traditional integer orders in integral and differential operators with arbitrary constant orders. Although it seems an elementary consideration, fractional order operators play an important role in describing many physical phenomena and have interesting implications. [1,2].

The introduction of the notion of variable-order (VO) integral and differential operators together with their some main properties was firstly initialized by Samko and Ross [3] in 1993. By these operators, one can define the order of the fractional integral and derivative as a function of independent variables such as time and space variables. In view of the characterization of the non-fixed kernel, this operators allows us to designate the memory and hereditary features of natural phenomena in a better way. By virtue of its potential efficiency to model real world problems, this topic has attracted many researchers in ongoing decades. In this direction, lots of papers have been published on different branches of science and engineering such as viscoelasticity, medicine, signal processing, control systems, so on [4 7]. Since its difficulty in getting an explicit solutions for fractional differential equations of VO, many papers have been devoted to find numerical solutions for this type of problems. See $[8-13]$ and the references cited therein. Hovewer very few paper on existence, uniquness and stability properties of fractional variable order differential equations have been published recently $14-20]$.

When we conduct an overview of the literature, increasing number of authors from several areas of the scientific community have focused on investigating the existence and uniqueness of fractional

*Corresponding Author 
constant order differential and integro-differential equations $[21-26]$.

In 27], Devi et al. studied the following Caputo's fractional boundary value problem by taking into consideration the monotone iterative technique.

$$
\left\{\begin{array}{l}
{ }^{C} D^{q} u=F\left(t, u, I^{q}(u)\right)+G\left(t, u, I^{q}(u)\right) \\
g(u(0), u(T))=0
\end{array}\right.
$$

where $0<q<1$. As a consequence, it has been shown that the established monotone flows converge uniformly to the coupled extremal solutions of the considered problem.

In [28, some sort of stability results were studied for fractional integro-differential equations involving Hilfer fractional derivative ${ }^{H} D_{a^{+}}^{\alpha, \beta ; \psi}$ (.) with $0<\alpha<1$ and $0 \leq \beta \leq 1$.

In particular, Bai and Kong 29 considered the existence of the solutions for the following initial value problem

$$
\left\{\begin{array}{l}
{ }^{C} D_{a^{+}}^{\alpha} y(t)=f\left(t, y(t), I_{a^{+}}^{\alpha} y(t)\right), t \in[a, b] \\
y(a)=x_{a}
\end{array}\right.
$$

by employing the upper and lower solution approach. The operators ${ }^{C} D_{0^{+}}^{\alpha}$ and $I_{0^{+}}^{\alpha}$ stand for the Caputo-Hadamard fractional derivative and Hadamard fractional integral operators of order $\alpha \in(0,1]$, respectively.

Motivated by the preceding works, we deal with the following boundary value problem on $\mathcal{J}:=$ $[0, b]$ such that

$$
\left\{\begin{array}{l}
{ }^{C} \mathcal{D}_{0^{+}}^{u(t)} y(t)=\Phi\left(t, y(t), I_{0^{+}}^{u(t)} y(t)\right) \\
y(0)=0, y(b)=0
\end{array}\right.
$$

where $1<u(t) \leq 2$ and ${ }^{C} \mathcal{D}_{0^{+}}^{u(t)}, I_{0^{+}}^{u(t)}$ are considered as in the sense of Caputo fractional derivative and integral of variable-order $u(t)$, respectively.

Our purpose is to investigate the existence and uniqueness of the solution of equation (1). We further show the stability of the solution in the Ulam-Hyers-Rassias (UHR) sense.

\section{Mathematical Preliminaries}

This part covers some fundamental concepts and lemmas that will be needed to understand the main theorems discussed in the subsequent sections.We also introduce some of the specifications for variable order operators.

Let $C(\mathcal{J}, \mathbb{R})$ denote the the set of all real-valued continuous functions from $\mathcal{J}$ into $\mathbb{R}$. For an element $\chi \in C(\mathcal{J}, \mathbb{R})$, define the standart norm $\|\chi\|=\operatorname{Sup}\{|\chi(t)|: t \in \mathcal{J}\}$, and with this norm $C(\mathcal{J}, \mathbb{R})$ becomes a Banach space.

For $-\infty<t_{1}<t_{2}<+\infty$, let the mappings to be defined $u(t):\left[t_{1}, t_{2}\right] \rightarrow(0,+\infty)$ and $v(t)$ : $\left[t_{1}, t_{2}\right] \rightarrow(n-1, n)$. Then, the left RiemannLioville(R-L) fractional integral of $\mathrm{VO} u(t)([30])$ is given as

$$
I_{t_{1}^{+}}^{u(t)} m(t)=\int_{t_{1}}^{t} \frac{(t-s)^{u(t)-1}}{\Gamma(u(t))} m(s) d s, t>t_{1},
$$

as well as the left VO Caputo derivative $([30)$ is defined by

$$
{ }^{C} \mathcal{D}_{t_{1}^{+}}^{v(t)} m(t)=\int_{t_{1}}^{t} \frac{(t-s)^{n-v(t)-1}}{\Gamma(n-v(t))} m^{(n)}(s) d s, t>t_{1} .
$$

These definitions, as expected, correspond with the usual R-L fractional integral and Caputo fractional derivative, respectively, when $u(t)$ and $v(t)$ are constant.see e.g. [3,30,31].

Lemma 1. ( 31]) Let $\sigma_{1}, \sigma_{2}>0, t_{1}>0$, $m \in L\left(t_{1}, t_{2}\right)$ and ${ }^{C} \mathcal{D}_{t_{1}^{+}} m \in L\left(t_{1}, t_{2}\right)$. Then, differential equation

$$
{ }^{C} \mathcal{D}_{t_{1}^{+}}^{\sigma_{1}} m(t)=0
$$

has the following general solution $m(t)=\alpha_{0}+\alpha_{1}\left(t-t_{1}\right)+\alpha_{2}\left(t-t_{1}\right)^{2}+\ldots+\alpha_{n-1}\left(t-t_{1}\right)^{n-1}$ where $n-1<\sigma_{1} \leq n$ and $\alpha_{\ell}(\ell=0,1, \ldots, n-1)$ are taken as arbitrary real numbers.

From that Lemma we deduce the next relation

$$
\begin{aligned}
I_{t_{1}^{+}}^{\sigma_{1} C} \mathcal{D}_{t_{1}^{+}}^{\sigma_{1}} m(t) & =m(t)+\alpha_{0}+\alpha_{1}\left(t-t_{1}\right) \\
& +\alpha_{2}\left(t-t_{1}\right)^{2}+\ldots+\alpha_{n-1}\left(t-t_{1}\right)^{n-1}
\end{aligned}
$$

Furthermore,

$$
{ }^{C} \mathcal{D}_{t_{1}^{+}}^{\sigma_{1}} I_{t_{1}^{+}}^{\sigma_{1}} m(t)=m(t) .
$$

and

$$
I_{t_{1}^{+}}^{\sigma_{1}} I_{t_{1}^{+}}^{\sigma_{2}} m(t)=I_{t_{1}^{+}}^{\sigma_{2}} I_{t_{1}^{+}}^{\sigma_{1}} m(t)=I_{t_{1}^{+}}^{\sigma_{1}+\sigma_{2}} m(t) .
$$

Remark 1. ( [32]) It's worth noting that the semigroup property isn't mostly satisfied by general functions $u(t), v(t)$, i.e.,

$$
I_{t_{1}^{+}}^{u(t)} I_{t_{1}^{+}}^{v(t)} m(t) \neq I_{t_{1}^{+}}^{u(t)+v(t)} m(t) .
$$

Definition 1. A function $\mu \in C(\mathcal{J}, \mathbb{R})$ is said to be a $C_{\delta}$ class function if it belong to the set

$$
C_{\delta}(\mathcal{J}, \mathbb{R})=\left\{\mu \in C((0, b], \mathbb{R}): t^{\delta} \mu \in C(\mathcal{J}, \mathbb{R})\right\}
$$

for $0 \leq \delta \leq 1$.

Lemma 2. [13] Assume that $u: \mathcal{J} \rightarrow(1,2)$ is a continuous function and $m \in C_{\delta}(\mathcal{J}, \mathbb{R})$. Then the fractional integral $I_{0^{+}}^{u(t)} m(t)$ of variable order exists for each point on $\mathcal{J}$.

Lemma 3. ([13]) Let $u \in C(\mathcal{J},(1,2))$ and $m \in C(\mathcal{J}, \mathbb{R})$ then $I_{0^{+}}^{u(t)} m(t) \in C(\mathcal{J}, \mathbb{R})$.

We now give the well-known Schauder fixed-point result. 
Theorem 1. ( [31]) Assume that $E$ is a Banach space and $Q$ is a nonempty convex subset of $E$ and moreover $F: Q \longrightarrow Q$ is compact, and continuous map. Then, there exist fixed points of $F$ in $Q$.

Definition 2. ([23]) The equation (1) is called Ulam-Hyers-Rassias (UHR) stable with respect to the function $\psi \in C\left(\mathcal{J}, \mathbb{R}^{+}\right)$if there exists $c_{\Phi}>0$, such that for any $\epsilon>0$ and for each solution $z \in C^{1}(\mathcal{J}, \mathbb{R})$ of the inequality $\left|{ }^{C} \mathcal{D}_{0^{+}}^{u(t)} z(t)-\Phi\left(t, z(t), I_{0^{+}}^{u(t)} z(t)\right)\right| \leq \epsilon \psi(t), t \in \mathcal{J}$, there exists a solution $y \in C(\mathcal{J}, \mathbb{R})$ of equation (1) with

$$
|z(t)-y(t)| \leq c_{\Phi} \epsilon \psi(t), t \in \mathcal{J} .
$$

\section{Existence Results}

Let us begin with introducing the following assumptions:

(H1): Let $\mathcal{P}=\left\{\mathcal{J}_{1}:=\left[0, b_{1}\right], \mathcal{J}_{2}:=\left(b_{1}, b_{2}\right], \mathcal{J}_{3}:=\right.$ $\left.\left(b_{2}, b_{3}\right], \ldots \mathcal{J}_{n}:=\left(b_{n-1}, b\right]\right\}$ be a partition of the interval $\mathcal{J}$, and let $u(t): \mathcal{J} \rightarrow(1,2]$ be a piecewise constant function with respect to $\mathcal{P}$, i.e.,

$$
u(t)=\sum_{\ell=1}^{n} u_{\ell} I_{\ell}(t)=\left\{\begin{array}{cl}
u_{1}, & \text { if } t \in \mathcal{J}_{1}, \\
u_{2}, & \text { if } t \in \mathcal{J}_{2}, \\
\cdot & \\
\cdot & \\
\cdot & \\
u_{n}, & \text { if } t \in \mathcal{J}_{n},
\end{array}\right.
$$

where $1<u_{\ell} \leq 2$ are constants, and $I_{\ell}$ is the indicator of the interval $\mathcal{J}_{\ell}:=\left(b_{\ell-1}, b_{\ell}\right]$,

$\ell=1,2, \ldots, n,\left(\right.$ with $\left.b_{0}=0, b_{n}=b\right)$ such that

$$
I_{\ell}(t)= \begin{cases}1, & \text { for } t \in \mathcal{J}_{\ell} \\ 0, & \text { for elsewhere. }\end{cases}
$$

(H2): Let $t^{\delta} \Phi: \mathcal{J} \times \mathbb{R} \times \mathbb{R} \rightarrow \mathbb{R}$ be a continuous function $(0 \leq \delta \leq 1)$, there exist constants $K, L>0$, satisfying the inequality

$$
\begin{aligned}
t^{\delta}\left|\Phi\left(t, w_{1}, z_{1}\right)-\Phi\left(t, w_{2}, z_{2}\right)\right| & \leq K\left|w_{1}-w_{2}\right| \\
& +L\left|z_{1}-z_{2}\right|,
\end{aligned}
$$

For each $\ell \in\{1,2, \ldots, n\}$, the set $E_{\ell}=C\left(\mathcal{J}_{\ell}, \mathbb{R}\right)$, represents the Banach space of continuous functions $y: \mathcal{J}_{\ell} \rightarrow \mathbb{R}$ equipped with the sup norm

$$
\|y\|_{E_{\ell}}=\sup _{t \in \mathcal{J}_{\ell}}|y(t)|,
$$

where $\ell \in\{1,2, \ldots, n\}$

We now analyze BVP defined in (1). On account of (3), the solution of (1) can be stated as

$$
\int_{0}^{t} \frac{(t-s)^{1-u(t)}}{\Gamma(2-u(t))} y^{\prime \prime}(s) d s=\Phi\left(t, y(t), I_{0^{+}}^{u(t)} y(t)\right),
$$

for $t \in \mathcal{J}$. If we employ $(H 1)$, the foregoing equation(4) can be written as

$$
\begin{aligned}
& \int_{0}^{b_{1}} \frac{(t-s)^{1-u_{1}}}{\Gamma\left(2-u_{1}\right)} y^{\prime \prime}(s) d s+\ldots \\
+ & \int_{b_{\ell-1}}^{t} \frac{(t-s)^{1-u_{\ell}}}{\Gamma\left(2-u_{\ell}\right)} y^{\prime \prime}(s) d s=\Phi\left(t, y(t), I_{0^{+}}^{u_{\ell}} y(t)\right)
\end{aligned}
$$

for $t \in \mathcal{J}_{\ell}, \ell=1,2, \ldots, n$.

The solution to the BVP (1) will be introduced in the following definition.

Definition 3. BVP (1) has a solution, if there are functions $y_{\ell}, \ell=1,2, \ldots, n$, such that $y_{\ell} \in$ $C\left(\left[0, b_{\ell}\right], \mathbb{R}\right)$ satisfying equation (5) and boundary conditions $y_{\ell}(0)=0=y_{\ell}\left(b_{\ell}\right)$.

Based on the preceding observation, BVP (1) can be represented as in (4) and, with considering $\mathcal{J}_{\ell}, \ell \in\{1,2, \ldots, n\}$ as in (5).

Since we define $y(t)$ identically 0 for $t \in\left[0, b_{\ell-1}\right)$, then the equation (5) is expressed as

$$
{ }^{C} \mathcal{D}_{b_{\ell-1}^{+}}^{u_{\ell}} y(t)=\Phi\left(t, y(t), I_{b_{\ell-1}^{+}}^{u_{\ell}} y(t)\right), \quad t \in \mathcal{J}_{\ell} .
$$

We shall deal with following BVP

$$
\left\{\begin{array}{l}
{ }^{C} \mathcal{D}_{b_{\ell-1}^{+}}^{u_{\ell}} y(t)=\Phi\left(t, y(t), I_{b_{\ell-1}^{+}}^{u_{\ell}} y(t)\right), \\
y\left(b_{\ell-1}\right)=0, y\left(b_{\ell}\right)=0,
\end{array}\right.
$$

for $t \in \mathcal{J}_{\ell}$. On the way to achieve our purpose, the upcoming lemma will play an important role.

Lemma 4. A function $y \in E_{\ell}$ establishes a solution for (6) if and only if $y$ fulfills the integral equation

$$
\begin{aligned}
y(t)= & -\frac{t-b_{\ell-1}}{b_{\ell}-b_{\ell-1}}\left[I_{b_{\ell-1}^{+}}^{u_{\ell}} \Phi\left(t, y(t), I_{b_{\ell-1}^{+}}^{u_{\ell}} y(t)\right)\right]_{t=b_{\ell}} \\
& +I_{b_{\ell-1}^{+}}^{u_{\ell}} \Phi\left(t, y(t), I_{b_{\ell-1}^{+}}^{u_{\ell}} y(t)\right) .
\end{aligned}
$$

Proof. We first assume that $y \in E_{\ell}$ is solution of the problem (6). If we apply the fractional operator $I_{b_{\ell-1}^{+}}^{u_{\ell}}$ to both sides of $\sqrt{6}$ and considering Lemma 1, we obtain

$$
\begin{aligned}
y(t)= & \alpha_{1}+\alpha_{2}\left(t-b_{\ell-1}\right)+\frac{1}{\Gamma\left(u_{\ell}\right)} \int_{b_{\ell-1}}^{t}(t-s)^{u_{\ell}-1} \\
& \times \Phi\left(s, y(s), I_{b_{\ell}^{+}}^{u_{\ell}} y(s)\right) d s,
\end{aligned}
$$

for $t \in \mathcal{J}_{\ell}$. By $y\left(b_{\ell-1}\right)=0$, we get $\alpha_{1}=0$.

Taking into account another boundary condition 
$y\left(b_{\ell}\right)=0$, it follows that

$$
\begin{aligned}
0= & \alpha_{2}\left(b_{\ell}-b_{\ell-1}\right) \\
+ & \frac{1}{\Gamma\left(u_{\ell}\right)} \int_{b_{\ell-1}}^{b_{\ell}}\left(b_{\ell}-s\right)^{u_{\ell}-1} \\
& \times \Phi\left(s, y(s), I_{b_{\ell-1}^{+}}^{u_{\ell}} y(s)\right) d s \\
\alpha_{2}= & -\left.\left(b_{\ell}-b_{\ell-1}\right)^{-1} I_{b_{\ell-1}^{+}}^{u_{\ell}} \Phi\left(t, y(t), I_{b_{\ell-1}^{+}}^{u_{\ell}} y(t)\right)\right|_{t=b_{\ell}}
\end{aligned}
$$

Then, we observe that

$$
\begin{aligned}
y(t)= & -\left(b_{\ell}-b_{\ell-1}\right)^{-1}\left(t-b_{\ell-1}\right) \\
& \times\left[I_{b_{\ell-1}^{+}}^{u_{\ell}} \Phi\left(t, y(t), I_{b_{\ell-1}^{+}}^{u_{\ell}} y(t)\right)\right]_{t=b_{\ell}} \\
+ & I_{b_{\ell-1}^{u_{\ell}}}^{u^{+}} \Phi\left(t, y(t), I_{b_{\ell-1}^{+}}^{u_{\ell}} y(t)\right), \quad t \in \mathcal{J}_{\ell} .
\end{aligned}
$$

On the contrary, let $y \in E_{\ell}$ be the solution of integral equation (7). Taking into account the continuity of function $t^{\delta} \Phi$ and using Lemma (1), we conclude that $y$ is the solution of the problem (6).

We can now show our first existence result which is based on Theorem (1)

Theorem 2. Assume that conditions (H1), (H2) hold, and if

$$
\frac{2\left(b_{\ell}-b_{\ell-1}\right)^{u_{\ell}-1}\left(b_{\ell}^{1-\delta}-b_{\ell-1}^{1-\delta}\right)}{(1-\delta) \Gamma\left(u_{\ell}\right)}\left(K+L \frac{\left(b_{\ell}-b_{\ell-1}\right)^{u_{\ell}}}{\Gamma\left(u_{\ell}+1\right)}\right)<1,
$$

then, there exist at least one solution for the problem (6) on $\mathcal{J}$.

Proof. Let us set the operator $W: E_{\ell} \rightarrow E_{\ell}$ such that for $t \in \mathcal{J}_{\ell}$

$$
\begin{aligned}
W y(t)= & -\left(b_{\ell}-b_{\ell-1}\right)^{-1}\left(t-b_{\ell-1}\right) \\
& \times\left.\left[I_{b_{\ell-1}^{+}}^{u_{\ell}} \Phi\left(t, y(t), I_{b_{\ell-1}^{+}}^{u_{\ell}} y(t)\right)\right]\right|_{t=b_{\ell}} \\
+ & \frac{1}{\Gamma\left(u_{\ell}\right)} \int_{b_{\ell-1}}^{t}(t-s)^{u_{\ell-1}} \\
& \times \Phi\left(s, y(s), I_{b_{\ell-1}^{+}}^{u_{\ell}} y(s)\right) d s .
\end{aligned}
$$

The operator $W: E_{\ell} \rightarrow E_{\ell}$ described in 3.1 is well defined, as seen by the properties of fractional integrals and the continuity of function $t^{\delta} \Phi$.

Let

$$
R_{\ell} \geq \frac{\frac{2 \eta_{0}\left(b_{\ell}-b_{\ell-1}\right)^{u_{\ell}}}{\Gamma\left(u_{\ell}\right)}}{1-\frac{2\left(b_{\ell}-b_{\ell-1}\right)^{u_{\ell}-1}\left(b_{\ell}^{1-\delta}-b_{\ell-1}^{1-\delta}\right)}{(1-\delta) \Gamma\left(u_{\ell}\right)}\left(K+L \frac{\left(b_{\ell}-b_{\ell-1}\right)^{u_{\ell}}}{\Gamma\left(u_{\ell}+1\right)}\right)},
$$

with

$$
\eta_{0}=\sup _{t \in \mathcal{J}_{\ell}}|\Phi(t, 0,0)|
$$

We generate the set

$$
B_{R_{\ell}}=\left\{y \in E_{\ell}:\|y\|_{E_{\ell}} \leq R_{\ell}\right\} .
$$

It is clear that $B_{R_{\ell}}$ is nonempty, closed, convex and bounded.
Now, we will see that $W$ satisfies the claims of the Theorem (1). We demonstrate it by using following stages.

STEP 1: We show that $W\left(B_{R_{\ell}}\right) \subseteq\left(B_{R_{\ell}}\right)$. For $y \in B_{R_{\ell}}$ and by (H2), we get$$
|W y(t)| \leq \frac{\left(b_{\ell}-b_{\ell-1}\right)^{-1}\left(t-b_{\ell-1}\right)}{\Gamma\left(u_{\ell}\right)}
$$

$$
\begin{aligned}
& \times \int_{b_{\ell-1}}^{b_{\ell}}\left(b_{\ell}-s\right)^{u_{\ell}-1} \\
& \times\left|\Phi\left(s, y(s), I_{b_{\ell-1}^{+}}^{u_{\ell}} y(s)\right)\right| d s \\
& +\frac{1}{\Gamma\left(u_{\ell}\right)} \int_{b_{\ell-1}}^{t}(t-s)^{u_{\ell}-1} \\
& \times\left|\Phi\left(s, y(s), I_{b_{\ell-1}^{+}}^{u_{\ell}} y(s)\right)\right| d s \\
& \leq \frac{2}{\Gamma\left(u_{\ell}\right)} \int_{b_{\ell-1}}^{b_{\ell}}\left(b_{\ell}-s\right)^{u_{\ell}-1} \\
& \times\left|\Phi\left(s, y(s), I_{b_{\ell-1}^{+}}^{u_{\ell}} y(s)\right)\right| d s \\
& =\frac{2}{\Gamma\left(u_{\ell}\right)} \int_{b_{\ell-1}}^{b_{\ell}}\left(b_{\ell}-s\right)^{u_{\ell}-1} \\
& \times\left|\Phi\left(s, y(s), I_{b_{\ell-1}^{+}}^{u_{\ell}} y(s)\right)-\Phi(s, 0,0)\right| d s \\
& +\frac{2}{\Gamma\left(u_{\ell}\right)} \int_{b_{\ell-1}}^{b_{\ell}}\left(b_{\ell}-s\right)^{u_{\ell}-1}|\Phi(s, 0,0)| d s \\
& \leq \frac{2}{\Gamma\left(u_{\ell}\right)} \int_{b_{\ell-1}}^{b_{\ell}}\left(b_{\ell}-s\right)^{u_{\ell}-1} s^{-\delta} \\
& \times\left(K|y(s)|+L\left|I_{b_{\ell-1}^{+}}^{u_{\ell}} y(s)\right|\right) d s \\
& +\frac{2 \eta_{0}\left(b_{\ell}-b_{\ell-1}\right)^{u_{\ell}}}{\Gamma\left(u_{\ell}\right)} \\
& \leq \frac{2\left(b_{\ell}-b_{\ell-1}\right)^{u_{\ell}-1}}{\Gamma\left(u_{\ell}\right)} \int_{b_{\ell-1}}^{b_{\ell}} s^{-\delta} \\
& \times\left(K+L \frac{\left(b_{\ell}-b_{\ell-1}\right)^{u} \ell}{\Gamma\left(u_{\ell}+1\right)}\right)|y(s)| d s \\
& +\frac{2 \eta_{0}\left(b_{\ell}-b_{\ell-1}\right)^{u_{\ell}}}{\Gamma\left(u_{\ell}\right)} \\
& \leq \frac{2\left(b_{\ell}-b_{\ell-1}\right)^{u_{\ell}-1}\left(b_{\ell}^{1-\delta}-b_{\ell-1}^{1-\delta}\right)}{(1-\delta) \Gamma\left(u_{\ell}\right)} \\
& \times\left(K+L \frac{\left(b_{\ell}-b_{\ell-1}\right)^{u_{\ell}}}{\Gamma\left(u_{\ell}+1\right)}\right) R_{\ell} \\
& +\frac{2 \eta_{0}\left(b_{\ell}-b_{\ell-1}\right)^{u_{\ell}}}{\Gamma\left(u_{\ell}\right)}
\end{aligned}
$$$$
\leq R_{\ell},
$$

which yields that $W\left(B_{R_{\ell}}\right) \subseteq B_{R_{\ell}}$.

STEP 2: $W$ is continuous.

We assume that the sequence $\left(y_{n}\right)$ converges to $y$ 
in $E_{\ell}$. Then,

$\left|\left(W y_{n}\right)(t)-(W y)(t)\right|$

$\leq \frac{\left(b_{\ell}-b_{\ell-1}\right)^{-1}\left(t-b_{\ell-1}\right)}{\Gamma\left(u_{\ell}\right)} \int_{b_{\ell-1}}^{b_{\ell}}\left(b_{\ell}-s\right)^{u_{\ell}-1}$

$\times\left|\Phi\left(s, y_{n}(s), I_{b_{\ell-1}^{+}}^{u_{\ell}} y_{n}(s)\right)-\Phi\left(s, y(s), I_{b_{\ell-1}^{+}}^{u_{\ell}} y(s)\right)\right| d s$

$+\frac{1}{\Gamma\left(u_{\ell}\right)} \int_{b_{\ell-1}}^{t}(t-s)^{u_{\ell}-1}$

$\times\left|\Phi\left(s, y_{n}(s), I_{b_{\ell-1}^{+}}^{u_{\ell}} y_{n}(s)\right)-\Phi\left(s, y(s), I_{b_{\ell-1}^{+}}^{u_{\ell}} y(s)\right)\right| d s$

$\leq \frac{2}{\Gamma\left(u_{\ell}\right)} \int_{b_{\ell-1}}^{b_{\ell}}\left(b_{\ell}-s\right)^{u_{\ell}-1}$

$\times\left|\Phi\left(s, y_{n}(s), I_{b_{\ell-1}^{+}}^{u_{\ell}} y_{n}(s)\right)-\Phi\left(s, y(s), I_{b_{\ell-1}^{+}}^{u_{\ell}} y(s)\right)\right| d s$

$\leq \frac{2}{\Gamma\left(u_{\ell}\right)} \int_{b_{\ell-1}}^{b_{\ell}} s^{-\delta}\left(b_{\ell}-s\right)^{u_{\ell}-1}$

$\left.\times\left(K\left|y_{n}(s)-y(s)\right|+L I_{b_{\ell-1}^{+}}^{u_{\ell}} \mid y_{n}(s)-y(s)\right) \mid\right) d s$

$\leq \frac{2 K}{\Gamma\left(u_{\ell}\right)}\left\|y_{n}-y\right\|_{E_{\ell}} \int_{b_{\ell-1}}^{b_{\ell}} s^{-\delta}\left(b_{\ell}-s\right)^{u_{\ell}-1} d s$

$+\frac{2 L}{\Gamma\left(u_{\ell}\right)}\left\|I_{b_{\ell-1}^{+}}^{u_{\ell}}\left(y_{n}-y\right)\right\|_{E_{\ell}} \int_{b_{\ell-1}}^{b_{\ell}} s^{-\delta}\left(b_{\ell}-s\right)^{u_{\ell}-1} d s$

$\leq \frac{2 K}{\Gamma\left(u_{\ell}\right)}\left\|y_{n}-y\right\|_{E_{\ell}} \int_{b_{\ell-1}}^{b_{\ell}} s^{-\delta}\left(b_{\ell}-s\right)^{u_{\ell}-1} d s$

$+\frac{2 L\left(b_{\ell}-b_{\ell-1}\right)^{u_{\ell}}}{\Gamma\left(u_{\ell}\right) \Gamma\left(u_{\ell}+1\right)}\left\|y_{n}-y\right\|_{E_{\ell}} \int_{b_{\ell-1}}^{b_{\ell}} s^{-\delta}\left(b_{\ell}-s\right)^{u_{\ell}-1} d s$

$\leq\left(\frac{2 K}{\Gamma\left(u_{\ell}\right)}+\frac{2 L\left(b_{\ell}-b_{\ell-1}\right)^{u_{\ell}}}{\Gamma\left(u_{\ell}\right) \Gamma\left(u_{\ell}+1\right)}\right)\left\|y_{n}-y\right\|_{E_{\ell}}$

$\times \int_{b_{\ell-1}}^{b_{\ell}} s^{-\delta}\left(b_{\ell}-s\right)^{u_{\ell}-1} d s$

$\leq \frac{2\left(b_{\ell}-b_{\ell-1}\right)^{u_{\ell}-1}\left(b_{\ell}{ }^{1-\delta}-b_{\ell-1}{ }^{1-\delta}\right)}{(1-\delta) \Gamma\left(u_{\ell}\right)}$

$\times\left(K+L \frac{\left(b_{\ell}-b_{\ell-1}\right)^{u_{\ell}}}{\Gamma\left(u_{\ell}+1\right)}\right)\left\|y_{n}-y\right\|_{E_{\ell}}$ i.e., we obtain

$\left\|\left(W y_{n}\right)-(W y)\right\|_{E_{\ell}} \rightarrow 0$ as $n \rightarrow \infty$.

As a consequence, the operator $W$ is a continuous on $E_{\ell}$.

STEP 3: $W$ is compact.

We will prove that $W\left(B_{R_{\ell}}\right)$ is relatively compact, which means that $W$ is compact. In view of step $1, W\left(B_{R_{\ell}}\right)$ is uniformly bounded. Namely, we have $W\left(B_{R_{\ell}}\right)=\left\{W(y): y \in B_{R_{\ell}}\right\} \subset B_{R_{\ell}}$ thus for each $y \in B_{R_{\ell}}$ we get $\|W(y)\|_{E_{\ell}} \leq R_{\ell}$ showing that $W\left(B_{R_{\ell}}\right)$ is bounded. Finally, It must be demonstrated the equicontinuity of $W\left(B_{R_{\ell}}\right)$.

For $t_{1}, t_{2} \in \mathcal{J}_{\ell}, t_{1}<t_{2}$ and $y \in B_{R_{\ell}}$, we write

$$
\left|(W y)\left(t_{2}\right)-(W y)\left(t_{1}\right)\right|
$$

$=\mid-\frac{\left(b_{\ell}-b_{\ell-1}\right)^{-1}\left(t_{2}-b_{\ell-1}\right)}{\Gamma\left(u_{\ell}\right)} \int_{b_{\ell-1}}^{b_{\ell}}\left(b_{\ell}-s\right)^{u_{\ell}-1}$

$\times \Phi\left(s, y(s), I_{b_{\ell-1}^{+}}^{u_{\ell}} y(s)\right) d s$

$+\frac{1}{\Gamma\left(u_{\ell}\right)} \int_{b_{\ell-1}}^{t_{2}}\left(t_{2}-s\right)^{u_{\ell}-1} \Phi\left(s, y(s), I_{b_{\ell-1}^{+}}^{u_{\ell}} y(s)\right) d s$

$$
\begin{aligned}
& +\frac{\left(b_{\ell}-b_{\ell-1}\right)^{-1}\left(t_{1}-b_{\ell-1}\right)}{\Gamma\left(u_{\ell}\right)} \int_{b_{\ell-1}}^{b_{\ell}}\left(b_{\ell}-s\right)^{u_{\ell}-1} \\
& \times \Phi\left(s, y(s), I_{b_{\ell-1}^{+}}^{u_{\ell}} y(s)\right) d s \\
& -\frac{1}{\Gamma\left(u_{\ell}\right)} \int_{b_{\ell-1}}^{t_{1}}\left(t_{1}-s\right)^{u_{\ell}-1} \Phi\left(s, y(s), I_{b_{\ell-1}^{+}}^{u_{\ell}} y(s)\right) d s \mid \\
& \leq \frac{\left(b_{\ell}-b_{\ell-1}\right)^{-1}}{\Gamma\left(u_{\ell}\right)}\left(\left(t_{2}-b_{\ell-1}\right)-\left(t_{1}-b_{\ell-1}\right)\right) \\
& \times \int_{b_{\ell-1}}^{b_{\ell}}\left(b_{\ell}-s\right)^{u_{\ell}-1}\left|\Phi\left(s, y(s), I_{b_{\ell-1}^{+}}^{u_{\ell}} y(s)\right)\right| d s \\
& +\frac{1}{\Gamma\left(u_{\ell}\right)} \int_{b_{\ell-1}}^{t_{1}}\left(\left(t_{2}-s\right)^{u_{\ell}-1}-\left(t_{1}-s\right)^{u_{\ell}-1}\right) \\
& \times\left|\Phi\left(s, y(s), I_{b_{\ell-1}^{+}}^{u_{\ell}} y(s)\right)\right| d s \\
& +\frac{1}{\Gamma\left(u_{\ell}\right)} \int_{t_{1}}^{t_{2}}\left(t_{2}-s\right)^{u_{\ell}-1}\left|\Phi\left(s, y(s), I_{b_{\ell-1}^{+}}^{u_{\ell}} y(s)\right)\right| d s \\
& \leq \frac{\left(b_{\ell}-b_{\ell-1}\right)^{-1}}{\Gamma\left(u_{\ell}\right)}\left(\left(t_{2}-b_{\ell-1}\right)-\left(t_{1}-b_{\ell-1}\right)\right) \\
& \times \int_{b_{\ell-1}}^{b_{\ell}}\left(b_{\ell}-s\right)^{u_{\ell}-1} \mid \Phi\left(s, y(s), I_{b_{\ell-1}^{+}}^{u_{\ell}} y(s)\right) \\
& \text { - } \Phi(s, 0,0) \mid d s \\
& +\frac{\left(b_{\ell}-b_{\ell-1}\right)^{-1}}{\Gamma\left(u_{\ell}\right)}\left(\left(t_{2}-b_{\ell-1}\right)-\left(t_{1}-b_{\ell-1}\right)\right) \\
& \times \int_{b_{\ell-1}}^{b_{\ell}}\left(b_{\ell}-s\right)^{u_{\ell}-1}|\Phi(s, 0,0)| d s \\
& +\frac{1}{\Gamma\left(u_{\ell}\right)} \int_{b_{\ell-1}}^{t_{1}}\left(\left(t_{2}-s\right)^{u_{\ell}-1}-\left(t_{1}-s\right)^{u_{\ell}-1}\right) \\
& \times\left|\Phi\left(s, y(s), I_{b_{\ell-1}^{+}}^{u_{\ell}} y(s)\right)-\Phi(s, 0,0)\right| d s \\
& +\frac{1}{\Gamma\left(u_{\ell}\right)} \int_{b_{\ell-1}}^{t_{1}}\left(\left(t_{2}-s\right)^{u_{\ell}-1}-\left(t_{1}-s\right)^{u_{\ell}-1}\right) \\
& \times|\Phi(s, 0,0)| d s+\frac{1}{\Gamma\left(u_{\ell}\right)} \int_{t_{1}}^{t_{2}}\left(t_{2}-s\right)^{u_{\ell}-1} \\
& \times\left|\Phi\left(s, y(s), I_{b_{\ell-1}^{+}}^{u_{\ell}} y(s)\right)-\Phi(s, 0,0)\right| d s \\
& +\frac{1}{\Gamma\left(u_{\ell}\right)} \int_{t_{1}}^{t_{2}}\left(t_{2}-s\right)^{u_{\ell}-1}|\Phi(s, 0,0)| d s \\
& \leq \frac{\left(b_{\ell}-b_{\ell-1}\right)^{-1}}{\Gamma\left(u_{\ell}\right)}\left(\left(t_{2}-b_{\ell-1}\right)-\left(t_{1}-b_{\ell-1}\right)\right) \\
& \times \int_{b_{\ell-1}}^{b_{\ell}}\left(b_{\ell}-s\right)^{u_{\ell}-1} \mid \Phi\left(s, y(s), I_{b_{\ell-1}^{+}}^{u_{\ell}} y(s)\right) \\
& \text { - } \Phi(s, 0,0) \mid d s \\
& +\frac{\left(b_{\ell}-b_{\ell-1}\right)^{-1}}{\Gamma\left(u_{\ell}\right)}\left(\left(t_{2}-b_{\ell-1}\right)-\left(t_{1}-b_{\ell-1}\right)\right) \\
& \times \int_{b_{\ell-1}}^{b_{\ell}}\left(b_{\ell}-s\right)^{u_{\ell}-1}|\Phi(s, 0,0)| d s \\
& +\frac{1}{\Gamma\left(u_{\ell}\right)} \int_{b_{\ell-1}}^{t_{1}}\left(\left(t_{2}-s\right)^{u_{\ell}-1}-\left(t_{1}-s\right)^{u_{\ell}-1}\right) \\
& \times\left|\Phi\left(s, y(s), I_{b_{\ell-1}^{+}}^{u_{\ell}} y(s)\right)-\Phi(s, 0,0)\right| d s \\
& +\frac{1}{\Gamma\left(u_{\ell}\right)} \int_{b_{\ell-1}}^{t_{1}}\left(\left(t_{2}-s\right)^{u_{\ell}-1}-\left(t_{1}-s\right)^{u_{\ell}-1}\right) \\
& \times|\Phi(s, 0,0)| d s+\frac{1}{\Gamma\left(u_{\ell}\right)} \int_{t_{1}}^{t_{2}}\left(t_{2}-s\right)^{u_{\ell}-1}
\end{aligned}
$$


$\times\left|\Phi\left(s, y(s), I_{b_{\ell-1}^{+}}^{u_{\ell}} y(s)\right)-\Phi(s, 0,0)\right| d s$

$+\frac{1}{\Gamma\left(u_{\ell}\right)} \int_{t_{1}}^{t_{2}}\left(t_{2}-s\right)^{u_{\ell}-1}|\Phi(s, 0,0)| d s$.

$\leq \frac{\left(b_{\ell}-b_{\ell-1}\right)^{-1}}{\Gamma\left(u_{\ell}\right)}\left(\left(t_{2}-b_{\ell-1}\right)-\left(t_{1}-b_{\ell-1}\right)\right)$

$\times \int_{b_{\ell-1}}^{b_{\ell}}\left(b_{\ell}-s\right)^{u_{\ell}-1} s^{-\delta}\left(K|y(s)|+L\left|I_{b_{\ell-1}^{+}}^{u_{\ell}} y(s)\right|\right) d s$

$+\frac{\eta_{0}\left(b_{\ell}-b_{\ell-1}\right)^{-1}}{\Gamma\left(u_{\ell}\right)}\left(\left(t_{2}-b_{\ell-1}\right)-\left(t_{1}-b_{\ell-1}\right)\right)$

$\times \int_{b_{\ell-1}}^{b_{\ell}}\left(b_{\ell}-s\right)^{u_{\ell}-1} d s$

$+\frac{1}{\Gamma\left(u_{\ell}\right)} \int_{b_{\ell-1}}^{t_{1}} s^{-\delta}\left(\left(t_{2}-s\right)^{u_{\ell}-1}-\left(t_{1}-s\right)^{u_{\ell}-1}\right)$

$\times\left(K|y(s)|+L\left|I_{b_{\ell-1}^{+}}^{u_{\ell}} y(s)\right|\right) d s$

$+\frac{\eta_{0}}{\Gamma\left(u_{\ell}\right)} \int_{b_{\ell-1}}^{t_{1}}\left(\left(t_{2}-s\right)^{u_{\ell}-1}-\left(t_{1}-s\right)^{u_{\ell}-1}\right) d s$

$+\frac{1}{\Gamma\left(u_{\ell}\right)} \int_{t_{1}}^{t_{2}} s^{-\delta}\left(t_{2}-s\right)^{u_{\ell}-1}$

$\times\left(K|y(s)|+L\left|I_{b_{\ell-1}^{+}}^{u_{\ell}} y(s)\right|\right) d s$

$+\frac{\eta_{0}}{\Gamma\left(u_{\ell}\right)} \int_{t_{1}}^{t_{2}}\left(t_{2}-s\right)^{u_{\ell}-1} d s$

$\leq \frac{\left(b_{\ell}-b_{\ell-1}\right)^{u_{\ell}-2}}{\Gamma\left(u_{\ell}\right)}\left(\left(t_{2}-b_{\ell-1}\right)-\left(t_{1}-b_{\ell-1}\right)\right)$

$\times\left(K\|y\|_{E_{\ell}}+L\left\|I_{b_{\ell-1}^{+}}^{u_{\ell}} y\right\|_{E_{\ell}}\right) \int_{b_{\ell-1}}^{b_{\ell}} s^{-\delta} d s$

$+\frac{\eta_{0}\left(b_{\ell}-b_{\ell-1}\right)^{u_{\ell}-1}}{\Gamma\left(u_{\ell}+1\right)}\left(\left(t_{2}-b_{\ell-1}\right)-\left(t_{1}-b_{\ell-1}\right)\right)$

$+\frac{1}{\Gamma\left(u_{\ell}\right)}\left(K\|y\|_{E_{\ell}}+L\left\|I_{b_{\ell-1}^{+}}^{u_{\ell}} y\right\|_{E_{\ell}}\right)$

$\times \int_{b_{\ell-1}}^{t_{1}} s^{-\delta}\left(\left(t_{2}-t_{1}\right)^{u_{\ell}-1}\right) d s$

$+\frac{\eta_{0}}{\Gamma\left(u_{\ell}\right)}\left(\frac{\left(t_{2}-b_{\ell-1}\right)^{u_{\ell}}}{u_{\ell}}-\frac{\left(t_{2}-t_{1}\right)^{u_{\ell}}}{u_{\ell}}-\frac{\left(t_{1}-b_{\ell-1}\right)^{u_{\ell}}}{u_{\ell}}\right)$

$+\frac{\left(t_{2}-t_{1}\right)^{u_{\ell}-1}}{\Gamma\left(u_{\ell}\right)}\left(K\|y\|_{E_{\ell}}+L\left\|I_{b_{\ell-1}^{+}}^{u_{\ell}} y\right\|_{E_{\ell}}\right) \int_{t_{1}}^{t_{2}} s^{-\delta} d s$

$+\frac{\eta_{0}}{\Gamma\left(u_{\ell}\right)} \frac{\left(t_{2}-t_{1}\right)^{u_{\ell}}}{u_{\ell}}$

$\leq \frac{\left(b_{\ell}-b_{\ell-1}\right)^{u_{\ell}-2}\left(b_{\ell}^{1-\delta}-b_{\ell-1}{ }^{1-\delta}\right)}{(1-\delta) \Gamma\left(u_{\ell}\right)}$

$\times\left(\left(t_{2}-b_{\ell-1}\right)-\left(t_{1}-b_{\ell-1}\right)\right)$

$\times\left(K\|y\|_{E_{\ell}}+L \frac{\left(b_{\ell}-b_{\ell-1}\right)^{u_{\ell}}}{\Gamma\left(u_{\ell}+1\right)}\|y\|_{E_{\ell}}\right)$

$+\frac{\eta_{0}\left(b_{\ell}-b_{\ell-1}\right)^{u_{\ell}-1}}{\Gamma\left(u_{\ell}+1\right)}\left(\left(t_{2}-b_{\ell-1}\right)-\left(t_{1}-b_{\ell-1}\right)\right)$

$+\left(\frac{\left(t_{1}^{1-\delta}-b_{\ell-1}{ }^{1-\delta}\right)\left(t_{2}-t_{1}\right)^{u_{\ell}-1}}{(1-\delta) \Gamma\left(u_{\ell}\right)}\right)$

$\times\left(K\|y\|_{E_{\ell}}+L \frac{\left(b_{\ell}-b_{\ell-1}\right)^{u_{\ell}}}{\Gamma\left(u_{\ell}+1\right)}\|y\|_{E_{\ell}}\right)+\frac{\eta_{0}}{\Gamma\left(u_{\ell}+1\right)}$

$\times\left(\left(t_{2}-b_{\ell-1}\right)^{u_{\ell}}-\left(t_{2}-t_{1}\right)^{u_{\ell}}-\left(t_{1}-b_{\ell-1}\right)^{u_{\ell}}\right)$

$+\frac{\left(t_{2}{ }^{1-\delta}-t_{1}{ }^{1-\delta}\right)\left(t_{2}-t_{1}\right)^{u_{\ell}-1}}{(1-\delta) \Gamma\left(u_{\ell}\right)}$

$$
\begin{aligned}
& \times\left(K\|y\|_{E_{\ell}}+L \frac{\left(b_{\ell}-b_{\ell-1}\right)^{u_{\ell}}}{\Gamma\left(u_{\ell}+1\right)}\|y\|_{E_{\ell}}\right)+\frac{\eta_{0}\left(t_{2}-t_{1}\right)^{u_{\ell}}}{\Gamma\left(u_{\ell}+1\right)} \\
& \leq\left(\frac{\left(b_{\ell}-b_{\ell-1}\right)^{u_{\ell}-2}\left(b_{\ell}{ }^{1-\delta}-b_{\ell-1}{ }^{1-\delta}\right)}{(1-\delta) \Gamma\left(u_{\ell}\right)}\left(K+L \frac{\left(b_{\ell}-b_{\ell-1}\right)^{u_{\ell}}}{\Gamma\left(u_{\ell}+1\right)}\right)\right. \\
& \left.\times\|y\|_{E_{\ell}}+\frac{\eta_{0}\left(b_{\ell}-b_{\ell-1}\right)^{u_{\ell}-1}}{\Gamma\left(u_{\ell}+1\right)}\right) \\
& \times\left(\left(t_{2}-b_{\ell-1}\right)-\left(t_{1}-b_{\ell-1}\right)\right) \\
& +\left(\frac{t_{2}{ }^{1-\delta}-b_{\ell-1}{ }^{1-\delta}}{(1-\delta) \Gamma\left(u_{\ell}\right)}\left(K+L \frac{\left(b_{\ell}-b_{\ell-1}\right)^{u_{\ell}}}{\Gamma\left(u_{\ell}+1\right)}\right)\|y\|_{E_{\ell}}\right) \\
& \times\left(t_{2}-t_{1}\right)^{u_{\ell}-1} \\
& +\frac{\eta_{0}}{\Gamma\left(u_{\ell}+1\right)}\left(\left(t_{2}-b_{\ell-1}\right)^{u_{\ell}}-\left(t_{1}-b_{\ell-1}\right)^{u_{\ell}}\right)
\end{aligned}
$$

Hence $\left\|(W y)\left(t_{2}\right)-(W y)\left(t_{1}\right)\right\|_{E_{\ell}} \rightarrow 0$ as $\left|t_{2}-t_{1}\right| \rightarrow$ 0 . It implies that $W\left(B_{R_{\ell}}\right)$ is equicontinuous.

As a consequence of the Theorem (1), the problem (6) has at least a solution $\widetilde{y_{\ell}}$ in $B_{R_{\ell}}$.

Let

$$
y_{\ell}=\left\{\begin{array}{l}
0, \quad t \in\left[0, b_{\ell-1}\right], \\
\widetilde{y}_{\ell}, t \in \mathcal{J}_{\ell},
\end{array}\right.
$$

We know that $y_{\ell} \in C\left(\left[0, b_{\ell}\right], X\right)$ defined by (8) satisfies the equation

$$
\begin{array}{r}
\int_{0}^{b_{1}} \frac{(t-s)^{1-u_{1}}}{\Gamma\left(2-u_{1}\right)} y_{\ell}^{\prime \prime}(s) d s+\ldots \\
+\int_{b_{\ell-1}}^{t} \frac{(t-s)^{1-u_{\ell}}}{\Gamma\left(2-u_{\ell}\right)} y_{\ell}^{\prime \prime}(s) d s=\Phi\left(t, y_{\ell}(t), I_{0^{+}}^{u_{\ell}} y_{\ell}(t)\right),
\end{array}
$$

for $t \in \mathcal{J}_{\ell}$, concluding that $y_{\ell}$ is a solution of (5) with $y_{\ell}(0)=0, y_{\ell}\left(b_{\ell}\right)=\widetilde{y}_{\ell}\left(b_{\ell}\right)=0$.

Then,

$$
y(t)=\left\{\begin{array}{l}
y_{1}(t), \quad t \in \mathcal{J}_{1}, \\
y_{2}(t)= \begin{cases}0, & t \in \mathcal{J}_{1}, \\
\widetilde{y}_{2}, & t \in \mathcal{J}_{2}\end{cases} \\
\cdot \\
\cdot \\
y_{n}(t)= \begin{cases}0, & t \in\left[0, b_{\ell-1}\right], \\
\widetilde{y}_{\ell}, & t \in \mathcal{J}_{\ell}\end{cases}
\end{array}\right.
$$

constitutes a solution for BVP(1).

The principle of Banach contraction is used to arrive at the following result.

Theorem 3. Assume that the assumptions (H1), (H2) hold and if

$$
\begin{array}{r}
\frac{2\left(b_{\ell}{ }^{1-\delta}-b_{\ell-1}{ }^{1-\delta}\right)\left(b_{\ell}-b_{\ell-1}\right)^{u_{\ell}-1}}{(1-\delta) \Gamma\left(u_{\ell}\right)} \\
\times\left(K+\frac{L\left(b_{\ell}-b_{\ell-1}\right)^{u_{\ell}}}{\Gamma\left(u_{\ell}+1\right)}\right)<1
\end{array}
$$

then the problem (6) has at most one solution in $E_{\ell}$. 
Proof. The Banach contraction concept will be used to demonstrate the unique fixed point for $W$ specified in Theorem (3).

For $y_{1}(t), y_{2}(t) \in E_{\ell}$, it follows that

$\left|\left(W y_{1}\right)(t)-\left(W y_{2}\right)(t)\right|$

$\leq \frac{\left(b_{\ell}-b_{\ell-1}\right)^{-1}\left(t-b_{\ell-1}\right)}{\Gamma\left(u_{\ell}\right)} \int_{b_{\ell-1}}^{b_{\ell}}\left(b_{\ell}-s\right)^{u_{\ell}-1}$

$\times\left|\Phi\left(s, y_{1}(s), I_{b_{\ell-1}^{+}}^{u_{\ell}} y_{1}(s)\right)-\Phi\left(s, y_{2}(s), I_{b_{\ell-1}^{+}}^{u_{\ell}} y_{2}(s)\right)\right| d s$

$+\frac{1}{\Gamma\left(u_{\ell}\right)} \int_{b_{\ell-1}}^{t}(t-s)^{u_{\ell}-1}$

$\times\left|\Phi\left(s, y_{1}(s), I_{b_{\ell-1}^{+}}^{u_{\ell}} y_{1}(s)\right)-\Phi\left(s, y_{2}(s), I_{b_{\ell-1}^{+}}^{u_{\ell}} y_{2}(s)\right)\right| d s$

$\leq \frac{2}{\Gamma\left(u_{\ell}\right)} \int_{b_{\ell-1}}^{b_{\ell}}\left(b_{\ell}-s\right)^{u_{\ell}-1}$

$\times\left|\Phi\left(s, y_{1}(s), I_{b_{\ell-1}^{+}}^{u_{\ell}} y_{1}(s)\right)-\Phi\left(s, y_{2}(s), I_{b_{\ell-1}^{+}}^{u_{\ell}} y_{2}(s)\right)\right| d s$

$\leq \frac{2}{\Gamma\left(u_{\ell}\right)} \int_{b_{\ell-1}}^{b_{\ell}}\left(b_{\ell}-s\right)^{u_{\ell}-1} s^{-\delta}$

$\left.\times\left(K\left|y_{1}(s)-y_{2}(s)\right|+L I_{b_{\ell-1}^{+}}^{u_{\ell}} \mid y_{1}(s)-y_{2}(s)\right) \mid\right) d s$

$\leq \frac{2 K}{\Gamma\left(u_{\ell}\right)}\left\|y_{1}-y_{2}\right\|_{E_{\ell}} \int_{b_{\ell-1}}^{b_{\ell}} s^{-\delta}\left(b_{\ell}-s\right)^{u_{\ell}-1} d s$

$+\frac{2 L}{\Gamma\left(u_{\ell}\right)}\left\|I_{b_{\ell-1}^{+}}^{u_{\ell}}\left(y_{1}-y_{2}\right)\right\|_{E_{\ell}} \int_{b_{\ell-1}}^{b_{\ell}} s^{-\delta}\left(b_{\ell}-s\right)^{u_{\ell}-1} d s$

$\leq \frac{2 K}{\Gamma\left(u_{\ell}\right)}\left\|y_{1}-y_{2}\right\|_{E_{\ell}} \int_{b_{\ell-1}}^{b_{\ell}} s^{-\delta}\left(b_{\ell}-s\right)^{u_{\ell}-1} d s$

$+\frac{2 L\left(b_{\ell}-b_{\ell-1}\right)^{u_{\ell}}}{\Gamma\left(u_{\ell}\right) \Gamma\left(u_{\ell}+1\right)}\left\|y_{1}-y_{2}\right\|_{E_{\ell}} \int_{b_{\ell-1}}^{b_{\ell}} s^{-\delta}\left(b_{\ell}-s\right)^{u_{\ell}-1} d s$

$\leq\left(\frac{2 K}{\Gamma\left(u_{\ell}\right)}+\frac{2 L\left(b_{\ell}-b_{\ell-1}\right)^{u_{\ell}}}{\Gamma\left(u_{\ell}\right) \Gamma\left(u_{\ell}+1\right)}\right)\left\|y_{1}-y_{2}\right\|_{E_{\ell}}$

$\times \int_{b_{\ell-1}}^{b_{\ell}} s^{-\delta}\left(b_{\ell}-s\right)^{u_{\ell}-1} d s$

$\leq \frac{2\left(b_{\ell}{ }^{1-\delta}-b_{\ell-1}{ }^{1-\delta}\right)\left(b_{\ell}-b_{\ell-1}\right)^{u_{\ell}-1}}{(1-\delta) \Gamma\left(u_{\ell}\right)}$

$\times\left(K+\frac{L\left(b_{\ell}-b_{\ell-1}\right)^{u_{\ell}}}{\Gamma\left(u_{\ell}+1\right)}\right)\left\|y_{1}-y_{2}\right\|_{E_{\ell}}$

Therefore, by considering (9), the operator $W$ is a contraction. Employing Banach contraction mapping, we result in that $W$ has only one fixed point, say it $\widetilde{y}_{\ell} \in E_{\ell}$, which also concludes that the problem (6) has got unique solution.

We let

$$
y_{\ell}=\left\{\begin{array}{l}
0, \quad t \in\left[0, b_{\ell-1}\right], \\
\widetilde{y}_{\ell}, \quad t \in \mathcal{J}_{\ell},
\end{array}\right.
$$

We know that $y_{\ell} \in C\left(\left[0, b_{\ell}\right], \mathbb{R}\right)$ defined by satisfies the equation

$$
\begin{array}{r}
\int_{0}^{b_{1}} \frac{(t-s)^{1-u_{1}}}{\Gamma\left(2-u_{1}\right)} y_{\ell}^{\prime \prime}(s) d s+\ldots \\
+\int_{b_{\ell-1}}^{t} \frac{(t-s)^{1-u_{\ell}}}{\Gamma\left(2-u_{\ell}\right)} y_{\ell}^{\prime \prime}(s) d s=\Phi\left(t, y_{\ell}(t), I_{0^{+}}^{u_{\ell}} y_{\ell}(t)\right),
\end{array}
$$

for $t \in \mathcal{J}_{\ell}$, which yields that $y_{\ell}$ is a unique solution of (5) with $y_{\ell}(0)=0, y_{\ell}\left(b_{\ell}\right)=\widetilde{y}_{\ell}\left(b_{\ell}\right)=0$.

This led us to

$$
y(t)=\left\{\begin{array}{l}
y_{1}(t), \quad t \in \mathcal{J}_{1}, \\
y_{2}(t)= \begin{cases}0, & t \in \mathcal{J}_{1}, \\
\widetilde{y}_{2}, & t \in \mathcal{J}_{2}\end{cases} \\
\cdot \\
\cdot \\
y_{n}(t)= \begin{cases}0, & t \in\left[0, b_{\ell-1}\right], \\
\widetilde{x}_{\ell}, & t \in \mathcal{J}_{\ell}\end{cases}
\end{array}\right.
$$

which is the unique solution of the boundary value problem (1).

\section{Ulam-Hyers-Rassias stability}

Theorem 4. Suppose that the conditions (H1), (H2), together with (9) hold. Assume further that

(H3): The function $\psi \in C\left(\mathcal{J}_{\ell}, \mathbb{R}_{+}\right)$have increasing property and there exists $\lambda_{\psi}>0$ such that

$$
I_{b_{\ell-1}+}^{u_{\ell}} \psi(t) \leq \lambda_{\psi} \psi(t)
$$

then, under these assumptions, the equation (1) has UHR stability with respect to $\psi$

Proof. Suppose that $z \in C\left(\mathcal{J}_{\ell}, \mathbb{R}\right)$ is a solution of the following inequality

$$
\left|{ }^{C} \mathcal{D}_{b_{\ell-1}{ }^{u_{\ell}}}^{u} z(t)-\Phi\left(t, z(t), I_{b_{\ell-1}{ }^{+}}^{u_{\ell}} z(t)\right)\right| \leq \epsilon \psi(t),
$$

for $t \in \mathcal{J}_{\ell}$. Let us denote $y \in C\left(\mathcal{J}_{\ell}, \mathbb{R}\right)$ to be the unique solution of the problem

$$
\left\{\begin{array}{l}
{ }^{C} \mathcal{D}_{b_{\ell-1}+}^{u_{\ell}} y(t)=\Phi\left(t, y(t), I_{b_{\ell-1}+}^{u_{\ell}} y(t)\right), t \in \mathcal{J}_{\ell} \\
y\left(b_{\ell-1}\right)=0, y\left(b_{\ell}\right)=0
\end{array}\right.
$$

By using Lemma (4), we have

$$
\begin{aligned}
y(t)= & -\frac{\left(b_{\ell}-b_{\ell-1}\right)^{-1}\left(t-b_{\ell-1}\right)}{\Gamma\left(u_{\ell}\right)} \int_{b_{\ell-1}}^{b_{\ell}}\left(b_{\ell}-s\right)^{u_{\ell-1}} \\
& \times \Phi\left(s, y(s), I_{b_{\ell-1}^{+}}^{u_{\ell}} y(s)\right) d s \\
+ & \frac{1}{\Gamma\left(u_{\ell}\right)} \int_{b_{\ell-1}}^{t}(t-s)^{u_{\ell-1}} \\
& \times \Phi\left(s, y(s), I_{b_{\ell-1}^{+}}^{u_{\ell}} y(s)\right) d s
\end{aligned}
$$


By integrating both sides of 11 and utilizing (H3), we find

$$
\begin{aligned}
& \mid z(t)+\frac{\left(b_{\ell}-b_{\ell-1}\right)^{-1}\left(t-b_{\ell-1}\right)}{\Gamma\left(u_{\ell}\right)} \\
& \times \int_{b_{\ell-1}}^{b_{\ell}}\left(b_{\ell}-s\right)^{u_{\ell-1}} \Phi\left(s, z(s), I_{b_{\ell-1}^{+}}^{u_{\ell}} z(s)\right) d s \\
& -\frac{1}{\Gamma\left(u_{\ell}\right)} \int_{b_{\ell-1}}^{t}(t-s)^{u_{\ell-1}} \Phi\left(s, z(s), I_{b_{\ell-1}^{+}}^{u_{\ell}} z(s)\right) d s \mid \\
& \leq \epsilon \int_{b_{\ell-1}}^{t} \frac{(t-s)^{u_{\ell}-1}}{\Gamma\left(u_{\ell}\right)} \psi(s) d s \\
& \leq \epsilon \lambda_{\psi} \psi(t)
\end{aligned}
$$

In addition, we get for each $t \in \mathcal{J}_{\ell}$

$$
\begin{aligned}
& |z(t)-y(t)| \\
& =\mid z(t)+\frac{\left(b_{\ell}-b_{\ell-1}\right)^{-1}\left(t-b_{\ell-1}\right)}{\Gamma\left(u_{\ell}\right)} \\
& \times \int_{b_{\ell-1}}^{b_{\ell}}\left(b_{\ell}-s\right)^{u_{\ell-1}} \Phi\left(s, y(s), I_{b_{\ell-1}^{+}}^{u_{\ell}} y(s)\right) d s \\
& -\frac{1}{\Gamma\left(u_{\ell}\right)} \int_{b_{\ell-1}}^{t}(t-s)^{u_{\ell-1}} \Phi\left(s, y(s), I_{b_{\ell-1}^{+}}^{u_{\ell}} y(s)\right) d s \mid \\
& \leq \mid z(t)+\frac{\left(b_{\ell}-b_{\ell-1}\right)^{-1}\left(t-b_{\ell-1}\right)}{\Gamma\left(u_{\ell}\right)} \\
& \times \int_{b_{\ell-1}}^{b_{\ell}}\left(b_{\ell}-s\right)^{u_{\ell-1}} \Phi\left(s, z(s), I_{b_{\ell-1}^{+}}^{u_{\ell}} z(s)\right) d s \\
& -\frac{1}{\Gamma\left(u_{\ell}\right)} \int_{b_{\ell-1}}^{t}(t-s)^{u_{\ell-1}} \Phi\left(s, z(s), I_{b_{\ell-1}^{+}}^{u_{\ell}} z(s)\right) d s \mid \\
& +\frac{\left(b_{\ell}-b_{\ell-1}\right)^{-1}\left(t-b_{\ell-1}\right)}{\Gamma\left(u_{\ell}\right)} \int_{b_{\ell-1}}^{b_{\ell}}\left(b_{\ell}-s\right)^{u_{\ell}-1} \\
& \times\left|\Phi\left(s, z(s), I_{b_{\ell-1}^{+}}^{u_{\ell}} z\right)-\Phi\left(s, y(s), I_{b_{\ell-1}^{+}}^{u_{\ell}} y\right)\right| d s \\
& +\frac{1}{\Gamma\left(u_{\ell}\right)} \int_{b_{\ell-1}}^{t}(t-s)^{u_{\ell}-1} \\
& \times\left|\Phi\left(s, z(s), I_{b_{\ell-1}^{+}}^{u_{\ell}} z\right)-\Phi\left(s, y(s), I_{b_{\ell-1}^{+}}^{u_{\ell}} y\right)\right| d s \\
& \leq \lambda_{\psi} \epsilon \psi(t) \\
& +\frac{\left(b_{\ell}-b_{\ell-1}\right)^{-1}\left(t-b_{\ell-1}\right)}{\Gamma\left(u_{\ell}\right)} \int_{b_{\ell-1}}^{b_{\ell}}\left(b_{\ell}-s\right)^{u_{\ell}-1} s^{-\delta} \\
& \times\left(K|z(s)-y(s)|+L I_{b_{\ell-1}^{+}}^{u_{\ell}}|z(s)-y(s)|\right) d s \\
& +\frac{1}{\Gamma\left(u_{\ell}\right)} \int_{b_{\ell-1}}^{t}(t-s)^{u_{\ell}-1} s^{-\delta} \\
& \times\left(K|z(s)-y(s)|+L I_{b_{\ell-1}^{+}}^{u_{\ell}}|z(s)-y(s)|\right) d s
\end{aligned}
$$

$$
\begin{aligned}
& \left.\leq \lambda_{\psi} \epsilon \psi(t)+\frac{\left(b_{\ell}-b_{\ell-1}\right)^{u_{\ell}-1}}{\Gamma\left(u_{\ell}\right)}\right) \\
& \times\left(K\|z-y\|_{E_{\ell}}+L\left\|I_{b_{\ell-1}^{+}}^{u_{\ell}}(z-y)\right\|_{E_{\ell}} \int_{b_{\ell-1}}^{b_{\ell}} s^{-\delta} d s\right. \\
& +\frac{\left(b_{\ell}-b_{\ell-1}\right)^{u_{\ell}-1}}{\Gamma\left(u_{\ell}\right)}\left(K\|z-y\|_{E_{\ell}}+L\left\|I_{b_{\ell-1}^{+}}^{u_{\ell}}(z-y)\right\|_{E_{\ell}}\right) \\
& \times \int_{b_{\ell-1}}^{t} s^{-\delta} d s \\
& \leq \lambda_{\psi} \epsilon \psi(t)+\frac{\left(b_{\ell}-b_{\ell-1}\right)^{u_{\ell}-1}\left(b_{\ell}^{1-\delta}-b_{\ell-1}{ }^{1-\delta}\right)}{(1-\delta) \Gamma\left(u_{\ell}\right)} \\
& \times\left(K\|z-y\|_{E_{\ell}}+L \frac{\left(b_{\ell}-b_{\ell-1}\right)^{u_{\ell}}}{\Gamma\left(u_{\ell}+1\right)}\|z-y\|_{E_{\ell}}\right) \\
& +\frac{\left(b_{\ell}-b_{\ell-1}\right)^{u_{\ell}-1}\left(t^{1-\delta}-b_{\ell-1}{ }^{1-\delta}\right)}{(1-\delta) \Gamma\left(u_{\ell}\right)} \\
& \times\left(K\|z-y\|_{E_{\ell}}+L \frac{\left(b_{\ell}-b_{\ell-1}\right)^{u_{\ell}}}{\Gamma\left(u_{\ell}+1\right)}\|z-y\|_{E_{\ell}}\right) \\
& \leq \lambda_{\psi} \epsilon \psi(t)+\frac{2\left(b_{\ell}-b_{\ell-1}\right)^{u_{\ell}-1}\left(b_{\ell}{ }^{1-\delta}-b_{\ell-1}{ }^{1-\delta}\right)}{(1-\delta) \Gamma\left(u_{\ell}\right)} \\
& \times\left(K+L \frac{\left(b_{\ell}-b_{\ell-1}\right)^{u} \ell}{\Gamma\left(u_{\ell}+1\right)}\right)\|z-y\|_{E_{\ell}}
\end{aligned}
$$

which gives

$$
\begin{aligned}
& \|z-y\|_{E_{\ell}} \\
& \times\left(1-\frac{2\left(b_{\ell}{ }^{1-\delta}-b_{\ell-1}{ }^{1-\delta}\right)\left(b_{\ell}-b_{\ell-1}\right)^{u_{\ell}-1}}{(1-\delta) \Gamma\left(u_{\ell}\right)}\right. \\
& \left.\times\left(K+L \frac{\left(b_{\ell}-b_{\ell-1}\right)^{u_{\ell}}}{\Gamma\left(u_{\ell}+1\right)}\right)\right) \\
& \leq \lambda_{\psi} \epsilon \psi(t)
\end{aligned}
$$

For each $t \in \mathcal{J}_{\ell}$, we arrive at the following relation

$$
\begin{aligned}
& \|z-y\|_{E_{\ell}} \\
& \leq \frac{\lambda_{\psi} \epsilon \psi(t)}{\left(1-\frac{2\left(b_{\ell}{ }^{1-\delta}-b_{\ell-1}{ }^{1-\delta}\right)\left(b_{\ell}-b_{\ell-1}\right)^{u_{\ell}-1}}{(1-\delta) \Gamma\left(u_{\ell}\right.}\left(K+L \frac{\left(b_{\ell}-b_{\ell-1}\right)^{u_{\ell}}}{\Gamma\left(u_{\ell}+1\right)}\right)\right.} \\
& =\left[1-\frac{2\left(b_{\ell}{ }^{1-\delta}-b_{\ell-1}{ }^{1-\delta}\right)\left(b_{\ell}-b_{\ell-1}\right)^{u_{\ell}-1}}{(1-\delta) \Gamma\left(u_{\ell}\right)}\right. \\
& \left.\times\left(K+L \frac{\left(b_{\ell}-b_{\ell-1}\right)^{u} \ell}{\Gamma\left(u_{\ell}+1\right)}\right)\right]^{-1} \lambda_{\psi} \epsilon \psi(t) \\
& :=c_{\Phi} \epsilon \psi(t)
\end{aligned}
$$

which concludes that the equation (6) admits UHR stability with respect to $\psi$ for each $i \in$ $\{1,2, \ldots, n\}$.

Consequently, main problem (1) has UHR stability with respect to $\psi$.

\section{Example}

Consider the fractional boundary value problem that follows: 


$$
\left\{\begin{array}{l}
{ }^{C} \mathcal{D}_{0^{+}}^{u(t)} y(t)=\frac{t^{-\frac{1}{3}} e^{-t}}{\left(e^{e^{\frac{t^{2}}{1+t}}}+4 e^{2 t}+1\right)\left(1+|y(t)|+\left|I_{0}^{u(t)} y(t)\right|\right)}, \\
y(0)=0, y(2)=0 .
\end{array}\right.
$$

for $t \in \mathcal{J}:=[0,2]$,

Let

$$
\Phi(t, y, z)=\frac{t^{-\frac{1}{3}} e^{-t}}{\left(e^{e^{\frac{t^{2}}{1+t}}}+4 e^{2 t}+1\right)(1+y+z)},
$$

$(t, y, z) \in[0,2] \times[0,+\infty) \times[0,+\infty)$ and

$$
u(t)= \begin{cases}\frac{3}{2}, & t \in \mathcal{J}_{1}:=[0,1], \\ \frac{9}{5}, & \left.\left.t \in \mathcal{J}_{2}:=\right] 1,2\right] .\end{cases}
$$

Then, we have

$$
\begin{aligned}
& t^{\frac{1}{3}}\left|\Phi\left(t, w_{1}, z_{1}\right)-\Phi\left(t, w_{2}, z_{2}\right)\right| \\
& =\left|\frac{e^{-t}}{\left(e^{\frac{t^{2}}{1+t}}+4 e^{2 t}+1\right)}\left(\frac{1}{1+w_{1}+z_{1}}-\frac{1}{1+w_{2}+z_{2}}\right)\right| \\
& \leq \frac{e^{-t}\left(\left|w_{1}-w_{2}\right|+\left|z_{1}-z_{2}\right|\right)}{\left(e^{\frac{t^{2}}{1+t}}+4 e^{2 t}+1\right)\left(1+w_{1}+z_{1}\right)\left(1+w_{2}+z_{2}\right)} \\
& \leq \frac{e^{-t}}{\left(e^{e^{\frac{t^{2}}{1+t}}}+4 e^{2 t}+1\right)}\left(\left|w_{1}-w_{2}\right|+\left|z_{1}-z_{2}\right|\right) \\
& \leq \frac{1}{(e+5)}\left|w_{1}-w_{2}\right|+\frac{1}{(e+5)}\left|z_{1}-z_{2}\right| .
\end{aligned}
$$

Let $\psi(t)=t^{\frac{1}{2}}$

$$
\begin{aligned}
I_{0^{+}}^{u_{1}} \psi(t) & =\frac{1}{\Gamma\left(\frac{3}{2}\right)} \int_{0}^{t}(t-s)^{\frac{1}{2}} s^{\frac{1}{2}} d s \\
& \leq \frac{1}{\Gamma\left(\frac{3}{2}\right)} \int_{0}^{t}(t-s)^{\frac{1}{2}} d s \\
& \leq \frac{2}{3 \Gamma\left(\frac{3}{2}\right)} \psi(t):=\lambda_{\psi} \psi(t) .
\end{aligned}
$$

It shows that the assumption (H3) holds with $\psi(t)=t^{\frac{1}{2}}$ and $\lambda_{\psi}=\frac{2}{3 \Gamma\left(\frac{3}{2}\right)}$.

Regarding Theorem (3), the problem (14) has a unique solution $y_{1} \in E_{1}$, and from Theorem (4) the solution of 14 is UHR stable.

For $t \in \mathcal{J}_{2}$, the problem $(12)$ can be written in the following way

$$
\left\{\begin{array}{l}
{ }^{C} \mathcal{D}_{1^{+}}^{\frac{9}{5}} y(t)=\frac{t^{-\frac{1}{3}} e^{-t}}{\left(e^{\frac{t^{2}}{1+t}}+4 e^{2 t}+1\right)\left(1+|y(t)|+\left|I_{0}^{9} y(t)\right|\right)}, \\
y(1)=0, \quad y(2)=0 .
\end{array}\right.
$$

We see that

$$
\begin{aligned}
& \frac{\left(b_{2}{ }^{1-\delta}-b_{1}{ }^{1-\delta}\right)\left(b_{2}-b_{1}\right)^{u_{2}-1}}{(1-\delta) \Gamma\left(u_{2}\right)}\left(2 K+\frac{2 L\left(b_{2}-b_{1}\right)^{u_{2}}}{\Gamma\left(u_{2}+1\right)}\right) \\
& =\frac{2^{\frac{2}{3}}-1}{\frac{2}{3} \Gamma\left(\frac{9}{5}\right)} \frac{2}{e+5}\left(1+\frac{1}{\Gamma\left(\frac{14}{5}\right)}\right) \simeq 0.3913<1
\end{aligned}
$$

Thus, the condition (9) is satisfied.

Also

$$
\begin{aligned}
I_{1^{+}}^{u_{2}} \psi(t) & =\frac{1}{\Gamma\left(\frac{9}{5}\right)} \int_{1}^{t}(t-s)^{\frac{4}{5}} s^{\frac{1}{2}} d s \\
& \leq \frac{1}{\Gamma\left(\frac{9}{5}\right)} \int_{1}^{t}(t-s)^{\frac{4}{5}} d s \\
& \leq \frac{5}{9 \Gamma\left(\frac{9}{5}\right)} \psi(t) \\
& :=\lambda_{\psi} \psi(t) .
\end{aligned}
$$

Therefore, the condition (H3) is satisfied with $\psi(t)=t^{\frac{1}{2}}$ and $\lambda_{\psi}=\frac{5}{9 \Gamma\left(\frac{9}{5}\right)}$.

Taking into account of Theorem (3), the problem (15) has a unique solution $\widetilde{y}_{2} \in E_{2}$, and from Theorem (4) the equation (15) has UHR stability.

It is known that

$$
y_{2}(t)=\left\{\begin{array}{l}
0, \quad t \in \mathcal{J}_{1} \\
\widetilde{y}_{2}(t), \quad t \in \mathcal{J}_{2} .
\end{array}\right.
$$

Hence, by considering definition (3), the boundary value problem 12 has got a unique solution

$$
y(t)=\left\{\begin{array}{l}
y_{1}(t), \quad t \in \mathcal{J}_{1}, \\
y_{2}(t)=\left\{\begin{array}{l}
0, \quad t \in \mathcal{J}_{1}, \\
\widetilde{y}_{2}(t), \quad t \in \mathcal{J}_{2} .
\end{array}\right.
\end{array}\right.
$$

Eventually, according to Theorem (4), the equation (12) is UHR stable with respect to $\psi$. 


\section{Conclusion}

We study some qualitative properties for a class of nonlinear fractional boundary value problems involving variable order operators. Since the existence and uniqueness as well as stability results to variable-order equations is rarely discussed in the literature, all of the outcomes in this paper have a great deal of potential for contributing to future researches.

\section{References}

[1] Baleanu, D., Machado, J. A. T., \& Luo, A. C. (Eds.). (2011). Fractional Dynamics and Control. Springer Science \& Business Media.

[2] Singh, H., Kumar, D., \& Baleanu, D. (Eds.). (2019). Methods of Mathematical Modelling: Fractional Differential Equations. CRC Press.

[3] Samko, S. G., \& Ross, B. (1993). Integration and differentiation to a variable fractional order. Integral Transforms and Special Functions, 1(4), 277-300.

[4] Gómez-Aguilar, J. F. (2018). Analytical and numerical solutions of a nonlinear alcoholism model via variable-order fractional differential equations. Physica A: Statistical Mechanics and its Applications, 494, 52-75.

[5] Sun, H., Chang, A., Zhang, Y., \& Chen, W. (2019). A review on variable-order fractional differential equations: mathematical foundations, physical models, numerical methods and applications. Fractional Calculus and Applied Analysis, 22(1), 27-59.

[6] Sun, H. G., Chen, W., Wei, H., \& Chen, Y. Q. (2011). A comparative study of constantorder and variable-order fractional models in characterizing memory property of systems. The European Physical Journal Special Topics, 193(1), 185-192.

[7] Sun, H., Chen, W., \& Chen, Y. (2009). Variable-order fractional differential operators in anomalous diffusion modeling. Physica A: Statistical Mechanics and its Applications, 388(21), 4586-4592.

[8] Akgül, A., \& Baleanu, D. (2017). On solutions of variable-order fractional differential equations. An International Journal of Optimization and Control: Theories $\&$ Applications (IJOCTA), 7(1), 112-116.

[9] Tavares, D., Almeida, R., \& Torres, D. F. (2016). Caputo derivatives of fractional variable order: numerical approximations. Communications in Nonlinear Science and $\mathrm{Nu}$ merical Simulation, 35, 69-87.

[10] Valério, D., \& Da Costa, J. S. (2011). Variable-order fractional derivatives and their numerical approximations. Signal Processing, 91(3), 470-483.

[11] Yang, J., Yao, H., \& Wu, B. (2018). An efficient numerical method for variable order fractional functional differential equation. Applied Mathematics Letters, 76, 221-226.

[12] Zhang, S., \& Hu, L. (2019). Unique existence result of approximate solution to initial value problem for fractional differential equation of variable order involving the derivative arguments on the half-axis. Mathematics, 7(3), 286.

[13] Zhang, S., Sun, S., \& Hu, L. (2018). Approximate solutions to initial value problem for differential equation of variable order. Journal of Fractional Calculus and Applications, 9(2), 93-112.

[14] Amar, B., Dumitru, B., Mohammed, S. S., Ali, H., \& Mustafa, I. (2021). Boundary value problem for nonlinear fractional differential equations of variable order via Kuratowski MNC technique. Advances in Difference Equations, 2021(1), 1-19.

[15] Zhang, S., \& Hu, L. (2019). The existence of solutions to boundary value problems for differential equations of variable order. Azerbaijan Journal of Mathematics, 9(1), 22-45.

[16] Benkerrouche, A., Souid, M. S., Chandok, S., \& Hakem, A. (2021). Existence and Stability of a Caputo Variable-Order Boundary Value Problem. Journal of Mathematics, 2021.

[17] Refice, A., Souid, M. S., \& Stamova, I. (2021). On the boundary value problems of Hadamard fractional differential equations of variable order via Kuratowski MNC technique. Mathematics, 9(10), 1134.

[18] Bouazza, Z., Souid, M. S., \& Gunerhan, H. (2021). Multiterm boundary value problem of Caputo fractional differential equations of variable order. Advances in Difference Equations, 2021(1), 1-17.

[19] Zhang, S. (2018). The uniqueness result of solutions to initial value problems of differential equations of variable-order. Revista de la Real Academia de Ciencias Exactas, Físicas y Naturales. Serie A. Matemáticas, 112(2), 407423.

[20] Amar, B., Souid, M. S., Kanokwan, S., \& Ali, H. (2021). Implicit nonlinear fractional differential equations of variable order. Boundary Value Problems, 2021(1).

[21] Yakar, A., \& Koksal, M. E. (2012). Existence results for solutions of nonlinear fractional differential equations. Abstract and Applied Analysis (Vol. 2012). Hindawi. 
[22] An, J., \& Chen, P. (2019). Uniqueness of solutions to initial value problem of fractional differential equations of variable-order. Dyn. Syst. Appl., 28, 607-623.

[23] Benchohra, M., \& Lazreg, J. E. (2017). Existence and Ulam stability for nonlinear implicit fractional differential equations with Hadamard derivative. Stud. Univ. BabesBolyai Math., 62(1), 27-38.

[24] Benchohra, M., \& Souid, M. S. (2015). L 1Solutions of boundary value problems for implicit fractional order differential equations. Surveys in Mathematics $\mathcal{E}$ its Applications, 10.

[25] Ashyralyev, A., \& Hicdurmaz, B. (2021). Multidimensional problems for nonlinear fractional Schrödinger differential and difference equations. Mathematical Methods in the Applied Sciences, 44(4), 2731-2751.

[26] Karakoç, F. (2020). Existence and uniqueness for fractional order functional differential equations with Hilfer derivative. Differ. Equ. Appl., 12, 323-336.

[27] Devi, J. V., \& Sreedhar, C. V. (2016). Generalized Monotone Iterative Method for Caputo Fractional Integro-differential Equation. European Journal of Pure and Applied Mathematics, 9(4), 346-359.

[28] de Oliveira, E. C., \& Sousa, J. V. D. C. (2018). Ulam-Hyers-Rassias stability for a class of fractional integro-differential equations. Results in Mathematics, 73(3), 1-16.
[29] Bai, Y., \& Kong, H. (2017). Existence of solutions for nonlinear Caputo-Hadamard fractional differential equations via the method of upper and lower solutions. J. Nonlinear Sci. Appl., 10(1), 5744-5752.

[30] Samko, S.G. (1995). Fractional integration and differentiation of variable order. Analysis Mathematica, 21(3), 213-236.

[31] Kilbas, A. A., Srivastava, H. M., \& Trujillo, J. J. (2006). Theory and Applications of Fractional Differential Equations. (Vol. 204). Elsevier.

[32] Zhang, S. (2013). Existence of solutions for two-point boundary-value problems with singular differential equations of variable order. Electronic Journal of Differential Equations, 2013(245), 1-16.

Ahmed Refice is a researcher from Djillali Liabes University of Sidi Bel-Abbès, Algeria

(10) https://orcid.org/0000-0002-8906-6211

Mohammed Said Souid is a professor at University of Tiaret, Algeria. He has many publications on existence and stability of fractional differential equations of variable order.

(10) https://orcid.org/0000-0002-4342-5231

Ali Yakar received his Ph.D.degree in applied mathematics from Gebze Institute of Technology, Turkey in 2010. His primary interest is in investigating existence and uniqueness of fractional order differential equations. He is currently working as a professor at Department of Mathematics at Tokat Gaziosmanpaşa University.

(10) https://orcid.org/0000-0003-1160-577X

An International Journal of Optimization and Control: Theories \& Applications (http://ijocta.balikesir.edu.tr)

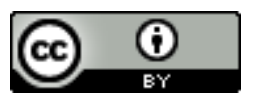

This work is licensed under a Creative Commons Attribution 4.0 International License. The authors retain ownership of the copyright for their article, but they allow anyone to download, reuse, reprint, modify, distribute, and/or copy articles in IJOCTA, so long as the original authors and source are credited. To see the complete license contents, please visit http://creativecommons.org/licenses/by/4.0/. 